\title{
Utilizing Intensity Ratios To Characterize Glass Fragments Via Micro-X-ray Fluorescence
}

\author{
Andrew H. Lee ${ }^{1}$ \\ 1. EDAX Inc., Mahwah, NJ USA
}

Micro-X-ray fluorescence (micro-XRF) is a non-destructive elemental technique which utilizes a focused X-ray source to generate characteristic radiation from the sample. In "bulk" samples, when the X-ray source cannot penetrate through the sample, the count-rates (CPS) for each element is saturated. Weight fractions can then be calculated by employing a Fundamental Parameters calibration model. However, if the sample is not thick enough to absorb the primary X-rays (i.e. "thin" sample), then the count-rates will be influenced by the sample thickness. This effects the calculation of weight fractions, as the Fundamental Parameters model assumes samples are infinitely thick. Figure 1 shows nominal Xray probe depths at various energies and samples matrices. These probe depths are crucial in determining if a sample can be considered "bulk" or "thin" relative to the X-ray energy. In many microXRF applications, comparing and characterizing small fragments (i.e. determining whether they are the same or different) is required. However, the samples may have no consistent thickness or size, and generally must be analyzed "as is". Therefore, a method is necessary to be able to compare samples while compensating for the influence of sample thickness. This study will look at an alternative intensity-based methodology to characterize samples, using measurements from soda-lime glass fragments to illustrate the improved stability.

In this method, weight fractions are ignored and instead, intensity ratios are used. Net intensities are calculated for each element, and then ratioed to the intensity of another element within the same sample. This is referred to as internal elemental ratios. It is based on the principle that while thickness will effect individual elemental intensities, the elements within that sample retain their relative ratios to each other (assuming consistent acquisition conditions).

Figure 2 shows two graphs of $\mathrm{Ca}(\mathrm{K})$ intensities measured in various soda-lime glass standards with varying thicknesses and varying levels of calcium. Normally for bulk samples, the correlation between $\mathrm{Ca}(\mathrm{K})$ intensities and their weight fractions would have been relatively linear. However, since these samples were inconsistently thin relative to the incoming X-rays, the correlation between net intensities against given concentrations is relatively unstable. The data points do not create a well-fit regression line $(\mathrm{R} 2=0.045)$ due to the influence of sample thickness. However, once $\mathrm{Ca}(\mathrm{K})$ intensities are normalized against the $\mathrm{Si}(\mathrm{K})$ intensity from the same sample, the correlation between the intensity ratios and their concentrations is significantly improved $(\mathrm{R} 2=0.8877)$.

This is critical in applications where glass characterization needs to be performed at a high degree of accuracy on thin samples, where unabsorbed X-rays can still penetrate through. By compensating for the influence of sample thickness, internal intensity ratios can provide improved correlation with elemental concentrations, while still allowing the sample to be analyzed non-destructively, with minimal preparation. 


\section{Analysis depths with $\mu$-EDXRF}

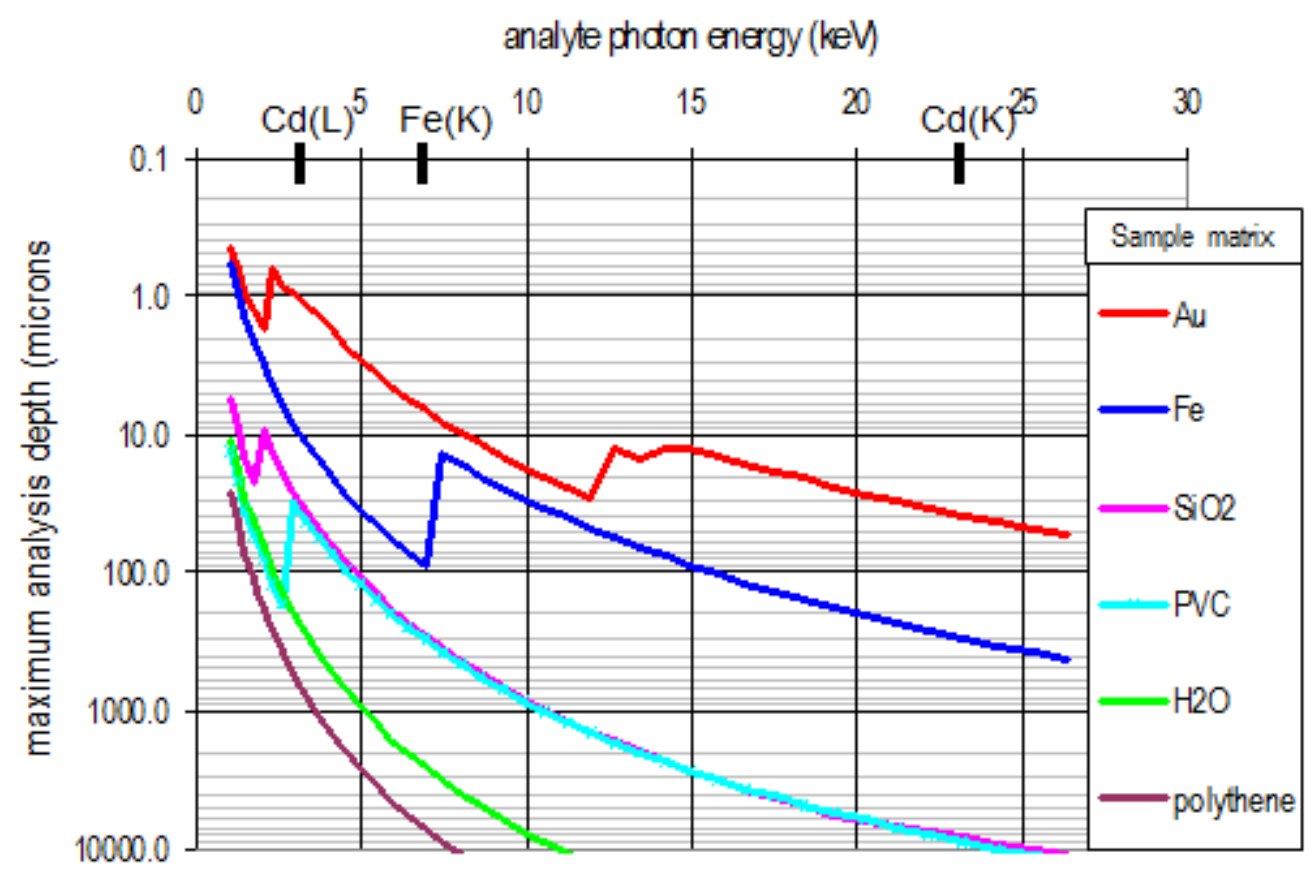

Figure 1. Nominal probe depths of X-rays in various sampling matrices. If the sample thickness does not exceed the analysis depth at a given energy, it is considered transparent to X-rays (i.e. thin sample).
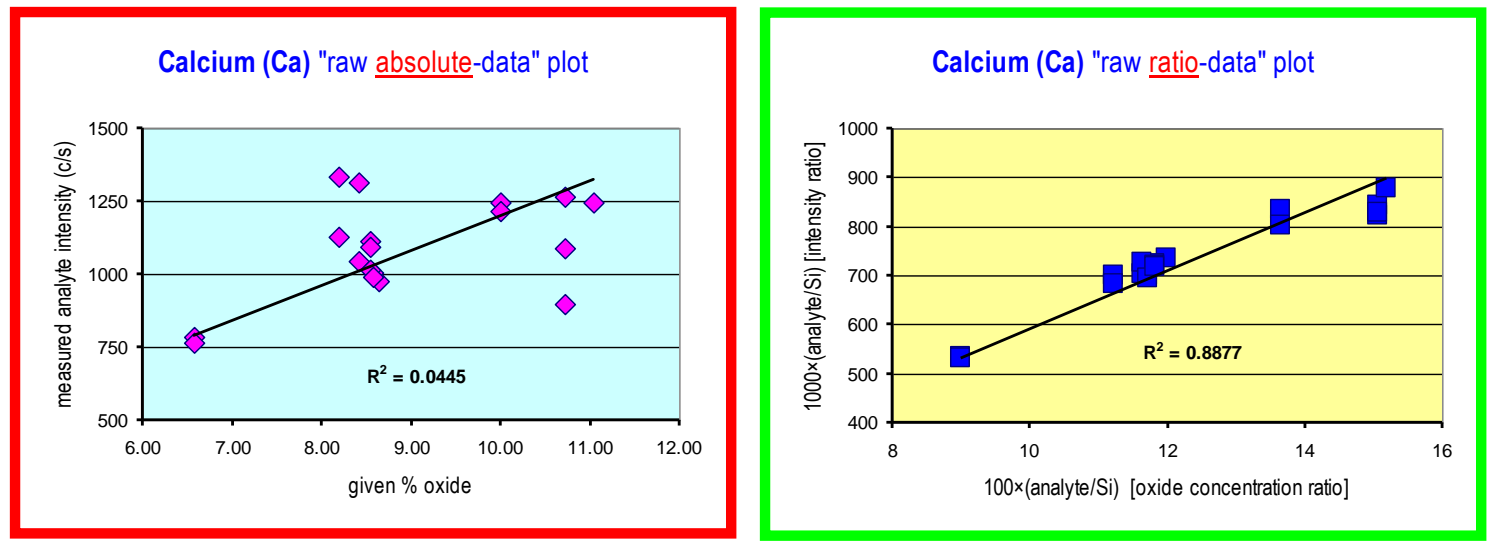

Figure 2. $\mathrm{Ca}(\mathrm{K})$ intensities compared, the first graph (L) shows raw net intensities compared to their reported weight fractions (\%). The influence of varying sample thickness creates a poorly fit regression line. Once the $\mathrm{Ca}(\mathrm{K})$ intensities were ratioed against $\mathrm{Si}(\mathrm{K})$ and re-plotted, their correlation to their reported weight fraction was substantially improved. 